\title{
Conditions for fast magnetic reconnection in astrophysical plasmas
}

\author{
M. Hanasz ${ }^{1}$ and H. Lesch ${ }^{2}$ \\ 1 Toruń Centre for Astronomy, Nicholas Copernicus University, 87148 Piwnice/Toruń, Poland \\ e-mail: mhanasz@astri.uni.torun.pl \\ ${ }^{2}$ University Observatory, München University, Scheinerstr. 1, 81679, Germany and Center for Interdisciplinary Plasma \\ Science (CIPS) \\ e-mail: lesch@usm.uni-muenchen.de
}

Received 27 November 2002 / Accepted 11 March 2003

\begin{abstract}
We investigate favourable circumstances for fast magnetic reconnection in astrophysical plasmas based on recent results by Rogers et al. (2001). Given that a critical magnetic field structure with antiparallel field lines exists, our analysis demonstrates that a sufficient condition for fast reconnection is that the ratio of the thermal pressure to the magnetic field pressure $\beta$ should be significantly larger than $2 m_{\mathrm{e}} / m_{\mathrm{p}}$ (twice the ratio of electron mass to proton mass). Using several examples (like the different components of the interstellar medium, the intergalactic medium, active galactic nuclei and jets) we show that in almost all astrophysical plasmas, magnetic reconnection proceeds fast i.e. independent of the resistivity, with a few percent of the Alfvén speed. Only for special cases like neutron stars and white dwarfs is $\beta$ smaller than $2 m_{\mathrm{e}} / m_{\mathrm{p}}$.
\end{abstract}

Key words. magnetohydrodynamics (MHD) - plasmas - magnetic fields

\section{Introduction}

As is well known, the process of magnetic reconnection is important in many space and astrophysical contexts. Since most astrophysical plasmas are magnetized, the process of magnetic reconnection is essential for the understanding of a broad variety of processes, like galactic and stellar dynamos, turbulence, particle acceleration and heating (e.g. Kulsrud 1999; Lesch 2000).

Two types of magnetic reconnection have been identified slow reconnection with inflow speeds significantly lower than the Alfvén velocity, and fast reconnection which proceeds with an inflow speed close to the Alfvén speed. The latter type of magnetic reconnection is the preferred one since it accounts for the fast timescale associated with solar flares, the solar magnetic cycle and the topological changes required for dynamo action in the interstellar medium of galaxies. The problem however is to understand how the fast reconnection can be realized given the physical parameters of most astrophysical plasmas. Since they are highly conducting, they are almost collisionless and can be treated as ideal magnetohydrodynamical systems in which magnetic reconnection cannot occur because of zero resistivity.

The question is what happens when magnetic field lines with antiparallel components encounter. This situation is typical for cosmic plasmas, since they are agitated by unsaturated external forces like differential rotation, winds, explosive motions or turbulence. In such nonequilibrium plasmas, at least in the initial stages, the kinetic energy density is typically much larger than the magnetic energy density, i.e. the kinetic pressure is higher than the magnetic pressure. The field lines react to these forces by being twisted, stretched and compressed, which easily leads to the encounter of antiparallel field lines, reaching magnetic energy densities comparable to the kinetic energy densities of the several external drivers. If this kind of dynamical equilibrium is reached, the magnetic field energy is transferred into current sheets in which the excess energy is dissipated by reconnection. In a way, reconnection is a relaxation mechanism unavoidable for any plasma which is externally distorted (Taylor 1986). Thus, magnetic reconnection is of fundamental importance for a deeper understanding of astrophysical plasmas.

The first model to describe such intersecting field lines was developed by Parker (1957) and Sweet (1958) in terms of enhanced magnetic diffusion in a layer with antiparallel field lines on both sides. Its principle is quite simple in terms of mass conservation: In steady state the magnetic diffusion velocity balances the incoming reconnection velocity whereas the plasma inflow across the sheet is balanced by plasma outflow along the layer. The plasma initially entrained on the magnetic field lines must escape from the reconnection zone. In the SweetParker scheme this means a bulk outflow parallel to the field lines within the layer. The condition that the plasma has to leave the reconnection zone is very important for the effectiveness of 
reconnection. The faster plasma expelled is from the layer, the higher inflow rate is allowing for a higher reconnection rate.

More quantitatively, starting from the assumption of stationary Ohmic dissipation in a three-dimensional reconnection sheet with an area $\sim L^{2}$ and a thickness $l$, the dissipation surface density in the sheet $l j^{2} \eta$ acts to reduce the influx of magnetic energy density $v_{\mathrm{r}} B^{2} / 8 \pi . v_{\mathrm{r}}$ is the approaching velocity of the field lines or reconnection speed and $\eta$ denotes the resistivity. Including mass conservation, Parker and Sweet calculated $v_{\mathrm{r}}$ to be of the order of $v_{\mathrm{r}} \simeq c_{\mathrm{A}} R m^{-1 / 2} . R m=c_{\mathrm{A}} L / \eta$ denotes the magnetic Reynolds number which is a large quantity in astrophysical plasmas (up to $10^{20}$ for the interstellar medium) and $c_{\mathrm{A}}$ is the Alfvèn speed. In other words, Sweet-Parker reconnection is very slow, too slow for any reasonable application in the solar photosphere or even the Galaxy.

Later, Petschek (1964) suggested a model in which shock waves open up the outflow channel allowing faster gas outflow and leading to a significantly faster inflow of field lines and thus faster reconnection speed. An X-point-like structure evolves in which in a localized region magnetic diffusion is fast. Outside that diffusion layer, shock waves accelerate the plasma leading to an open X-point structure. Petschek obtained a reconnection speed of about $c_{\mathrm{A}} / \ln R m$, much faster than the Sweet-Parker value and almost independent of the Reynolds number. The Petschek model is known as fast reconnection. However, it has been shown (Biskamp 1986, 1996; Uzdensky \& Kulsrud 2000) that the Petschek solution is not compatible with uniform or smooth profiles of the electrical resistivity $\eta$.

MHD reconnection corresponds to localized current sheets in which, due to some resistivity, the energy density $\eta j^{2}$ is dissipated. It is the value and spatial profile of electrical resistivity $\eta$ which is the unknown in astrophysical plasmas. Normally, $\eta$ is produced by Coulomb collisions, i.e. $\eta \simeq T^{-3 / 2}$. Thus, most astrophysical plasmas are collisionless, i.e. $\eta \sim 0$ with respect to Coulomb collisions. This is also the reason why the magnetic Reynolds number $R m \propto 1 / \eta$ is so large. This is the key question for MHD reconnection.

On the other hand, $\eta$ can be enhanced due to plasma microinstabilities which are often excited only in the reconnection regions where free energy is available either in the form of a large drift between ions and electrons or in strong pressure and magnetic field gradients. This anomalous resistivity not only broadens the current sheet thereby increasing the mass inflow and the reconnection rate in the context of the SweetParker model (Kulsrud 2001) but also its localization is able to open up the outflow channel for the fast reconnection (Sato \& Hayashi 1979; Biskamp \& Schwarz 2001). Alternatively, a recent theory (Rogers et al. 2001), attempts to explain fast reconnection rates based on non-dissipative terms, notably the Hall term in the generalized Ohm's law. When the physics of reconnection is associated with the Hall term as was recently shown by Rogers et al. (2001) the value of resistivity (if there is any) is not at all crucial. Nevertheless, any reconnection process requires that some critical gradient has been exceeded by the magnetic field structure to offer the necessary amount of free energy fed into plasma fluctuations. Only if such a critical magnetic field gradient, i.e. a critical current density, has been exceeded, magnetic reconnection can start and proceed. The question is what is the reconnection speed?

The question of operation of the fast reconnection in astrophysical plasmas is of primary importance. Its presence would resolve some problems related to turbulent dynamos, as well as energetics of stellar coronae, accretion and galactic disks (Kulsrud \& Anderson 1992; Kulsrud 1999).

It is the aim of this paper to investigate the consequences of recent findings in collisionless plasma simulations, namely that magnetic reconnection is almost always fast in astrophysical plasmas. In the next section we briefly summarize the results of the simulations. Then we transform the conditions by Rogers et al. (2001) in order to make them applicable for astrophysical conditions. Finally, we apply these results to astrophysical systems and present some conclusions.

\section{Plasma simulations of collisionless magnetic reconnection}

Recently, several two-fluid and particle simulations have revealed fast rates of magnetic reconnection that significantly exceed those of conventional resistive magnetohydrodynamic models (Birn et al. 2001). Such high reconnection rates depend sensitively on the formation of an open X-line (as was already suggested by Petschek 1964), i.e. the thickness of the reconnection layer has to increase with distance from the X-point (Shay et al. 1998). It is the small-scale dynamics that provide the fast reconnection dynamics. Small scales mean the electron skin depth $d_{\mathrm{e}}=c / \omega_{\mathrm{pe}}$ and ion skin depth $d_{\mathrm{i}}=c / \omega_{\mathrm{pi}}$, respectively. Here $\omega_{\text {pe }}$ denotes the electron plasma frequency and $\omega_{\mathrm{pi}}$ is the ion plasma frequency. Furthermore, at these small scales the electrons decouple from the ions. Electrons are strongly magnetized and their flow scales inversely with the width of the layer. When the layer shrinks the electrons are accelerated. This leads to an electron flux from the layer which remains large although the reconnection layer size decreases. Of course the ions follow and the plasma is expelled with high speeds from the reconnection region. The reconnection rate becomes insensitive to the mechanism that is responsible for the nonidealness (Rogers et al. 2001).

In Fig. 1 we illustrate the 3-D geometry of the magnetic field in Cartesian coordinates in the vicinity of the current sheet. Before the onset of reconnection the sheet is coplanar with the $x z$-plane. The $y$-direction is perpendicular to the current sheet.

The plasma $\beta$ parameter is related to the total magnetic field $\boldsymbol{B}$ and the angle $\theta$ denotes the inclination of $\boldsymbol{B}$ with respect to the $x$-axis. $B_{x}=B \cos \theta$ is the reconnecting component of the magnetic field and $B_{z}=B \sin \theta$ is the guiding field. The $B_{y}$ component is zero prior to the reconnection event. It becomes finite as a result of reconnection.

The dynamics of the reconnection layer have been reproduced by simulations of Rogers et al. (2001) that show that the combined action of whistler waves and kinetic Alfvén waves play the central role in producing the open outflow region what characterizes the two-fluid and particle simulations. Both waves obey dispersion relations $\omega \propto k^{2}$, i.e. their phase velocities $v_{\phi} \propto k$ increase with decreasing spatial scale. For whistler 


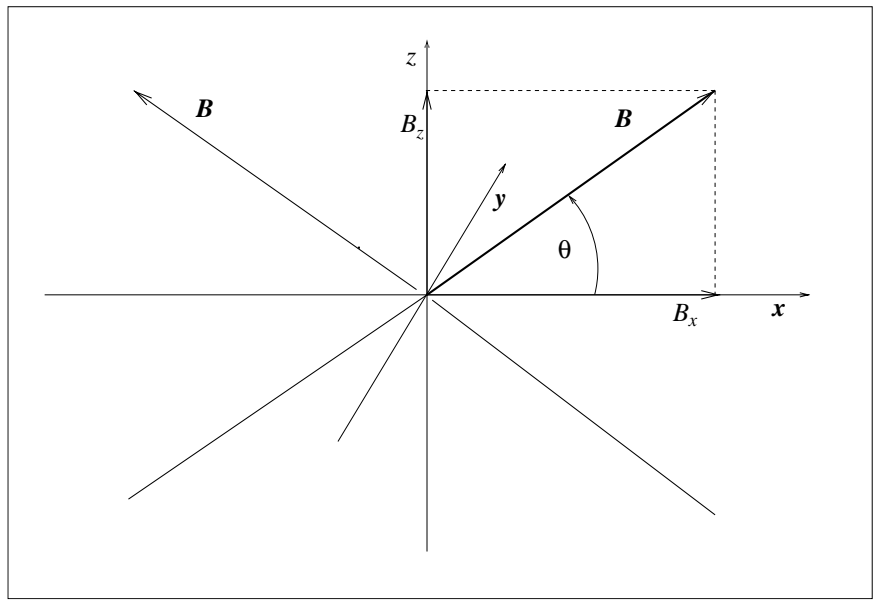

Fig. 1. Geometry of the magnetic field in the vicinity of the current sheet before the onset of reconnection. The current sheet corresponding to the contact discontinuity of magnetic field is coplanar with the $x z$ plane. The two magnetic field vectors inclined with respect to the $x$-axis represent two magnetic field lines on both sides of the current sheet.

waves the dispersion relation is $\omega=k^{2} c_{\mathrm{Ak}} d_{\mathrm{i}}$. Kinetic Alfvén waves obey the relation $\omega=k r_{\mathrm{gi}} k_{\|} c_{\mathrm{A}}$, where $k_{\|}$denotes the wave vector parallel to the magnetic field.

Rogers et al. (2001) show that the dynamics of reconnection is related to the presence of whistler and kinetic Alfvén waves that is controlled by two dimensionless parameters:

$\frac{\beta_{\mathrm{k}}}{2}=\frac{c_{\mathrm{s}}^{2}}{c_{\mathrm{Ak}}^{2}}$

$\mu_{\mathrm{k}}=\frac{c_{m}^{2}}{c_{\mathrm{Ak}}^{2}} \frac{m_{\mathrm{e}}}{m_{\mathrm{i}}}$

where $c_{\mathrm{s}}^{2}=k_{\mathrm{B}}\left(T_{\mathrm{e}}+T_{\mathrm{i}}\right) / m_{\mathrm{i}}, c_{\mathrm{Ak}}^{2}=(\boldsymbol{B} \cdot \boldsymbol{k} / k)^{2} /(4 \pi \rho), c_{m}^{2}=c_{\mathrm{A}}^{2}+c_{\mathrm{s}}^{2}$ and $c_{\mathrm{A}}^{2}=B^{2} /(4 \pi \rho)$. $\beta_{\mathrm{k}}$ and $\mu_{\mathrm{k}}$ measure the strength of the guiding magnetic field and the plasma pressure. The two-parameter space $\left(\beta_{\mathrm{k}}, \mu_{\mathrm{k}}\right)$ splits into four regimes (Fig. 2 in Rogers et al. 2001) admitting different combinations of whistler and kinetic Alfvén waves:

1. $\mu_{\mathrm{k}} \ll 1, \beta_{\mathrm{k}} / 2 \gg 1$ - both whistler and kinetic Alfvén waves possible.

2. $\mu_{\mathrm{k}} \ll 1, \beta_{\mathrm{k}} / 2 \leq 1-$ only whistler waves possible

3. $\mu_{\mathrm{k}} \geq 1, \beta_{\mathrm{k}} / 2 \gg \mu_{\mathrm{k}}-$ only kinetic Alfvén waves possible.

4. $\mu_{\mathrm{k}} \geq 1, \beta_{\mathrm{k}} / 2 \leq \mu_{\mathrm{k}}-$ no quadratic dispersive waves.

The numerical simulations presented by Rogers et al. (2001) demonstrate that the condition of fast magnetic reconnection of the Petschek type is related to the existence of dispersive waves of the two types mentioned above, i.e. fast magnetic reconnection operates in the first three regimes. On the other hand, only the absence of dispersive waves in regime 4 allows for the slow Parker-Sweet model.

The results of numerical simulations are interpreted in terms of wave analysis. The fast reconnection is possible when the X-type pattern of separatrices is stationary. Its existence is equivalent to the presence of a stationary magnetic field component $B_{y}$ perpendicular to the reconnecting component $B_{x 0}$.
The magnitude of the $B_{y}$ component, which is limited to $B_{x 0}$, is not known a priori since there is no $B_{y}$ in the initial state and later on $B_{y}$ appears as a result of reconnection.

The transition from the initial state (before the onset of reconnection) to the appearance of a finite $B_{y}$ component has to be time-dependent, although this dependence is unknown. Rogers et al. (2001) discuss conditions for the mentioned two types of dispersive waves propagating in the $y$ direction $(\boldsymbol{k}=$ $k \hat{e}_{y}$ ). They consider first the parameters $\beta_{y}$, and $\mu_{y}$, both depending on $B_{y}$, thus unknown functions of time. They find a relation between $\mu_{y}$ and $\beta_{y} / 2 . B_{x}$ is vanishing at the $x z$-plane for $y=0$, thus

$\mu_{y}=\frac{c_{\mathrm{A} y}^{2}+c_{\mathrm{A} z}^{2}+c_{s}^{2}}{c_{\mathrm{A} y}^{2}} \frac{m_{\mathrm{e}}}{m_{\mathrm{i}}}$

can be expressed as

$\mu_{y}=\frac{m_{\mathrm{e}}}{m_{\mathrm{i}}}+C \frac{\beta_{y}}{2} \simeq C \frac{\beta_{y}}{2}$,

where $C=m_{\mathrm{e}} / m_{\mathrm{i}}\left(1+2 / \beta_{z}\right)$ and $\beta_{z}=c_{\mathrm{s}}^{2} / c_{\mathrm{A} z}^{2}$ is the plasma $\beta$ associated with the guiding field $B_{z}$. Equation (4) describes a trajectory of the reconnection process in the $\left(\mu_{y}, \beta_{y}\right)$-parameter space for an unknown time dependence of the trajectory. It turns out, according to conditions $1-4$, that if $C<1$, the whole trajectory lies in the regimes 1,2 and 3 where the fast reconnection operates. The constant $C$ parametrizes the magnitude of the guiding field. The condition $C<1$ is equivalent to the statement that the guiding field is below a certain critical value corresponding to $\beta_{z}=2 m_{\mathrm{e}} / m_{\mathrm{i}}$, and $C>1$ means that the critical guiding field is exceeded. In the latter case the trajectory lies only partially in region 2 corresponding to whistler waves $\left(\mu_{y}<1\right)$. The remaining part of the trajectory is placed in region 4 which does not admit dispersive waves. Therefore the condition for fast reconnection $\mu_{y}<1$ implies that

$\frac{\beta_{y}}{2} \simeq \frac{1}{C}$

i.e. a certain threshold of $B_{y}$ has to be exceeded. However, the vanishing $B_{y}$ at $t=0$ places the initial configuration just in region 4.

Therefore $\beta_{z}$ should not be too small to initiate the fast reconnection,

$\beta_{z} \geq \frac{2 m_{\mathrm{e}}}{m_{\mathrm{i}}}$,

which is equivalent to the statement that the guiding field should not be too strong.

The condition (6) is a sufficient, but not necessary, condition for fast reconnection. This means that if condition (6) is met, fast reconnection will occur. But this does not mean that fast reconnection will not occur if condition (6) is not met. In particular, large fluctuations or small-scale instabilities (which typically occur in a narrow current layer) could lead to a large enough $B_{y}$ so as to initiate fast reconnection.

Although the last mentioned effects provide an opportunity for fast reconnection in the regime $\beta_{z}<2 m_{\mathrm{e}} / m_{\mathrm{i}}$, we cannot 
quantify them on theoretical ground nor detect them observationally in astrophysical systems. For that reason we treat the condition (6) as a safe limitation of the fast reconnection process in the parameter space.

The conditions (1) and (2) for whistler and kinetic Alfvén wave dynamics can be expressed in terms of parameters describing of the medium far away from the current sheet or parameters before the onset of reconnection.

Rogers et al. (2001) conclude that: "The condition for whistler dynamics to be present,

$B_{x}^{2}>B^{2}\left(1+\frac{\beta}{2}\right) \frac{m_{\mathrm{e}}}{m_{\mathrm{i}}}$,

is satisfied in many system of physical interest. Assuming this condition is satisfied, the physics at the smallest scales $\sim c / \omega_{\text {pe }}$ characteristic for the dissipation region are always governed by whistler waves". On the other hand "if plasma beta is sufficiently high

$\frac{\beta}{2} \geq \max \left[\left(1+\frac{\beta}{2}\right) \frac{m_{\mathrm{e}}}{m_{\mathrm{i}}}, \frac{B_{x}^{2}}{B^{2}}\right]$,

then kinetic Alfvén dynamics also play a role at somewhat larger scales".

\section{The conditions for fast reconnection in randomly distorted astrophysical plasmas}

In order to apply the findings by Rogers et al. (2001) to astrophysical plasmas we note that with the exception of the earth magnetosphere, where in-situ satellite measurements are possible, the relevant spatial scales of individual reconnection events are much too small to be spatially resolved by any astrophysical observation. This means that an evaluation of the conditions for fast reconnection is not possible for individual current sheets in distant astrophysical objects. The only way is to investigate the parameter space $\left(\beta_{\mathrm{k}}, \mu_{\mathrm{k}}\right)$ in different variables $(\beta, \theta)$, where $\beta$ is observable and $\theta$ is an individual property of each current sheet. Since $\theta$ is not available from observations, the evaluation of conditions for fast reconnection in astrophysical objects is not possible, unless we incorporate additional knowledge about the systems under consideration.

This necessary additional piece of information has to rely on the fact that even in cases of very strong magnetic fields a full range of angles $\theta$ can be expected (in a statistical sense) due to the fact that a huge reservoir of gravitational potential energy is available for random distortions of magnetic field lines. Therefore $\theta$ can be treated as a random variable. In such a case the determination whether fast reconnection is possible or not depends on the existence of a range of angles $\theta$ admitting reconnection. If such a range for given $\beta$ exists, fast reconnection is possible. Thus the two dimensional parameter space can be reduced to a one dimensional space of plasma- $\beta$. In the following considerations we perform such a transformation of the parameter space.

The conditions (7) and (8) can be expressed in terms of two parameters: plasma- $\beta$ and the pitch angle $\theta$. The conditions for whistler wave dynamics and kinetic Alfvén wave dynamics are

$\left(1+\frac{\beta}{2}\right) \frac{m_{\mathrm{e}}}{m_{\mathrm{i}}}<\cos ^{2} \theta$

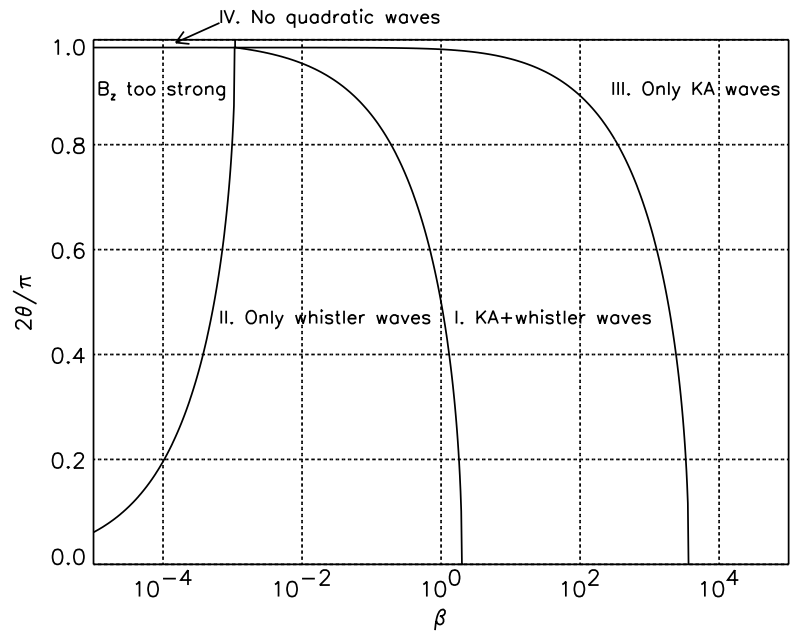

Fig. 2. Parameter space for quadratic waves. Numbering of different regions follows the list following conditions (1) and (2). An additional region on the left of the figure results form the upper limit on the guiding field $B_{z}$.

and

$\frac{\beta}{2}>\max \left[\left(1+\frac{\beta}{2}\right) \frac{m_{\mathrm{e}}}{m_{\mathrm{i}}}, \cos ^{2} \theta\right]$,

respectively.

The condition (6) for an upper limit for the magnitude of the guiding magnetic field can be analogously written in the form

$\frac{\beta}{2}>\frac{m_{\mathrm{e}}}{m_{\mathrm{i}}} \sin ^{2} \theta$.

The conditions above for dispersive wave dynamic expressed in terms of parameters $(\beta, \theta)$ are presented in Fig. 2.

The division of the parameter space shown in Fig. 2 is basically the same as displayed in Fig. 2 of Rogers et al. (2001). The only difference is that we divided region II (admitting only whistler waves) into two subregions following the condition (11) resulting from the upper limit of the guiding magnetic field.

The aim of the modified parametrization of the results by Rogers et al. (2001) is to determine the range of pitch angles $\theta$ admitting fast reconnection for a given value of plasma- $\beta$. We will subsequently implement the new formulation of the conditions for fast reconnection to a turbulent medium.

Keeping in mind the fact that we consider small regions of a medium disturbed by external forces we can assume that the distribution of pitch angles $\theta$ is uniform in the range [0, $\pi / 2]$. Therefore, one can expect fast reconnection for a given value of plasma- $\beta$ even if a narrow range of pitch angles fulfills the conditions for fast reconnection. We note however that efficiency of the reconnection will be dependent on the widths of the range of appropriate pitch angles.

We note that both kinds of dispersive waves operate for a wide range of pitch angles (excluding cases of a very weak reconnecting component $B_{x}$ ) for plasma- $\beta$ between one and several $10^{3}$. This range is extremely important for astrophysical plasmas. For $\beta$ above several $10^{3}$ fast reconnection is associated with kinetic Alfvén waves. For $\beta$ varying in between $2 m_{\mathrm{e}} / m_{\mathrm{i}}$ 
Table 1. Parameters and plasma $\beta$ for various astrophysical objects.

\begin{tabular}{lcccc}
\hline \hline Type of medium & $\begin{array}{c}\text { Density } \\
\mathrm{cm}^{-3}\end{array}$ & Mag. field & $\begin{array}{c}\text { Temp } \\
\mathrm{K}\end{array}$ & Plasma $\beta$ \\
Unit & $10^{-6}$ & $0.5 \mu \mathrm{G}$ & $10^{7}$ & 0.14 \\
\hline Gas in supercusters of gal. & $10^{-4}$ & $1 \mu \mathrm{G}$ & $10^{7}$ & 3.5 \\
Gas in clusters of gal. & $10^{-3}$ & $4 \mu \mathrm{G}$ & $10^{6}$ & 0.22 \\
Gas in galactic halos & 0.5 & $4 \mu \mathrm{G}$ & $10^{4}$ & 1.09 \\
Spiral arm interstellar gas. & 0.8 & $4 \mu \mathrm{G}$ & $10^{3}$ & 0.3 \\
Large interstellar shells-initial & 3 & $5 \mu \mathrm{G}$ & 100 & 0.04 \\
HI gas in diffuse clouds & 5 & $10 \mu \mathrm{G}$ & $10^{4}$ & 1.74 \\
HII regions & 90 & $15 \mu \mathrm{G}$ & 100 & 0.14 \\
HI gas in interclumps & 100 & $15 \mu \mathrm{G}$ & 10 & 0.02 \\
HI gas in abs. initial & $10^{17}$ & $2 \times 10^{3} \mathrm{G}$ & $10^{4}$ & 0.8 \\
Solar photosphere (spots) & $10^{8}$ & $10 \mathrm{G}$ & $10^{6}$ & $3.5 \times 10^{-3}$ \\
Solar corona & $10^{10}$ & $10^{3}-10^{4} \mathrm{G}$ & $10^{6}$ & $3 \times 10^{-5}$ \\
Magnetic stars & $10^{3}$ & $10^{-4} \mathrm{G}$ & 10 & $3 \times 10^{-3}$ \\
Bipolar flows & $10^{10}$ & $10^{-3}-10^{-1} \mathrm{G}$ & $10^{6}-10^{8}$ & 0.3 \\
AGN nuclei & $10^{-4}$ & $10^{-5}-10^{-3} \mathrm{G}$ & $10^{6}-10^{7}$ & $10^{-2}$ \\
AGN jets & $10^{12}$ & $10^{12} \mathrm{G}$ & $2 \times 10^{6}$ & $10^{-20}$ \\
Neutron star surface & $10^{12}$ & $10^{6} \mathrm{G}$ & $10^{6}$ & $10^{-8}$ \\
Magnetized white dwarfs & & & & \\
\hline
\end{tabular}

and 1 the whistler wave dynamics contributes to fast reconnection and moreover there is a range of large pitch angles admitting operation of both kinds of dispersive waves.

Considering the lowest values of plasma- $\beta$ up to $2 m_{\mathrm{e}} / m_{\mathrm{i}}$ we note that only a narrow range of pitch angles (region IV) does not admit quadratic waves, however the adjacent region of smaller pitch angles requires large fluctuations or other types of small scale instabilities to exceed a threshold in $B_{y}$ for strong guiding fields $B_{z}$. Fast reconnection is still possible due to whistler waves at very low plasma- $\beta$ only if the field lines on opposite sides of the current sheet are almost antiparallel.

Now we shall discuss astrophysical consequences of the above findings. The most common astrophysical circumstance is a turbulent medium that is agitated by external forces and/or instabilities. The natural behaviour of relaxing MHD systems is the spontaneous formation of current sheets. The encountering regions with non-parallel magnetic fields are filled with magnetic fields of typical strengths which is, in principle, an observable quantity. On the other hand the geometry of field lines around the contact discontinuity is usually unknown. Therefore, only the typical magnitude of plasma $\beta$ can be estimated for a particular system.

Thus, we can conclude that the conditions for fast magnetic reconnection in a turbulent medium can be related to the typical magnitude of plasma $\beta$ in the medium considered.

\section{Dynamics of astrophysical plasmas}

In Table 1 (partially from Vallée 1995 and from Tajima \& Shibata 1997) we present the most important plasma parameters: particle density, magnetic field strength and temperature for several astrophysical object classes. With these values we can estimate the value of $\beta=8 \pi n k_{\mathrm{B}} T / B^{2}$. Obviously, only for neutron stars, magnetized white dwarfs and in magnetic stars the ratio of thermal energy density to magnetic energy density is significantly smaller than one. For plasmas like the ionized gas in superclusters, in galaxy clusters, in galactic halos, in spiral arms, the neutral HI gas in interclumps and the ionized gas in HII regions, the plasma beta is of the order of one or somewhat larger.

Combining these numbers with the results from completely different reconnection simulations, we can conclude that in almost every astrophysical plasma, magnetic reconnection proceeds at a fast rate.

What is the physical reason for the distribution of $\beta$ ? The answer is that the magnetic fields in cosmic plasmas are the result of some processes which convert kinetic plasma energy into magnetic energy. The kinetic energy of a cosmic plasma is the result of the different forces acting on the ionized gas.

Of course, the most important force in the universe is gravity, but in many systems like stellar accretion disks, disks around black holes, planetary systems and disk galaxies, it is rotation which at least temporarily balances gravity. This balance results in differentially rotating plasmas, i.e. shear flows. Other sources of shear flows are stellar winds, stellar explosions and jets. In molecular clouds it is a mixture of turbulent motions and shear flows that dominate the plasma dynamics. In any case, it is the kinetic energy density $1 / 2 \rho v^{2}$ which represents the ultimate source of the magnetic field energy. The velocity $v$ may be due to turbulence, rotation or a directed large-scale flow. Such external unsaturated forces like 
differential rotation, gravity and/or explosions, winds and jets agitate the magnetized plasmas. That is, the amplification of magnetic fields is equivalent to a transfer of plasma kinetic energy into magnetic energy. Therefore, magnetic fields can hardly grow to field strengths larger than equipartition value given by $1 / 2 \rho v^{2}=B^{2} / 8 \pi$. In a virialized plasma we can reasonably expect equipartition between kinetic energy density and and thermal energy density $n k_{\mathrm{B}} T$, as well. In non steady plasmas, kinetic energy will dominate over the magnetic energy density.

There are two possibilities for deviations from these rather general arguments:

1. The frozen-in magnetic field is under the influence of gravitational collapse of a stellar object, like in the case of white dwarfs and neutron stars. There the plasma pressure is negligible compared to the gravitational potential, i.e. the magnetic field strength is increasing as the size of the system shrinks due to gravitational collapse which is stopped by the pressure of the degenerated stellar material. Since stars are spheres, they prefer dipolar magnetic field configurations that connect the star with its environment by a magnetosphere. When the rigidly rotating magnetospheres reach the Alfvén speed the magnetic field decouples from the stellar field and winds up into a solar wind-like spiral configuration which is at some distance in equipartition with the pressures in the remnant region.

2. Only for small spatial scales the magnetic field energy density can be significantly larger than the thermal energy density. That happens in stellar coronae and photospheres. There, the gas density drops faster than the magnetic field strengths. Due to footpoint motions of the stellar surface the magnetic field lines that escape into the photospheres or coronae represent an energy density that may be higher than the thermal energy density. But as we know from the Sun, even in the solar corona the plasma beta is not as small as that required to make reconnection proceed at a slow rate. On the contrary, the fine structure and the energy requirements of solar flares were some of the major arguments supporting fast reconnection. To understand very rapid flares, reconnection must be very fast. In the solar corona the plasma $\beta$ is small but still, fast reconnection is at work. This supports for the recent findings of plasma simulation groups.

\section{Conclusions}

We have applied the results of the most sophisticated up to date simulations of magnetic reconnection to astrophysical plasmas. There is a long-standing discussion as to whether reconnection proceeds slowly (according to the Sweet-Parker mechanism) with a velocity significantly smaller than the Alfvén speed, or fast with a few percent of the Alfvén speed. A definite answer to this problem is very important to understand dynamo action in stars and galaxies, as well as for the understanding of stellar flares. All these phenomena require the action of fast reconnection.

First, we emphasize that magnetic reconnection can only proceed if field lines with antiparallel directions are close enough. In other words, only if some critical value for the current density has been exceeded is enough free energy is available for the excitation of plasma fluctuations necessary to induce enhanced resistivity. In other words, fast reconnection can start after the current sheets appear. However, formation of current sheets may introduce an additional time delay for the onset of reconnection. This time delay should be rather attributed to macroscopic properties of the system and may be responsible for the criticality of the reconnection phenomena even in the case of plasma $\beta$ of the order of one.

Recently a number of plasma simulations demonstrated that magnetic reconnection should be fast in almost every circumstance provided the critical field structure has been established by external forces acting on the magnetic field lines. Especially the results of Rogers et al. (2001) lead to the conclusion that reconnection is unconditionally fast if the magnetic field strength is not too high i.e. if $\beta \geq 2 m_{\mathrm{e}} / m_{\mathrm{p}}$. For $\beta<2 m_{\mathrm{e}} / m_{\mathrm{p}}$, fast reconnection is conditionally possible except in a narrow range of pitch angles close to $\pi / 2$ (region IV of small reconnecting magnetic field component) if large fluctuations or small-scale instabilities could lead to a large enough $B_{y}$ or the pitch angle $\theta$ is sufficiently small (near antiparallel magnetic fields on both sides of the current sheet).

If the field is not so strong, reconnection is fast and its rate does not depend on the resistivity. Lazarian \& Vishniac (2000) already concluded from their investigations of turbulent magnetic reconnection that this process becomes fast when field stochasticity is taken into account. As a consequence solar and galactic dynamos are also fast, i.e. do not depend on fluid resistivity. We support their conclusions by checking the betavalues for different astrophysical systems. Only for the very strongly magnetized stellar remnants (neutron stars and magnetized white dwarfs) the possibility of fast reconnection depends on additional conditions listed above.

In stellar photospheres, where the beta-values are also small but larger than $2 m_{\mathrm{e}} / m_{\mathrm{p}}$, the magnetic field is still not rigid enough to inhibit fast reconnection, as is proven by the fast solar flares. For all other cosmical plasmas, reconnection will proceed at a rate comparable to the Alfvén speed.

From our investigation we can conclude that especially in weak magnetic fields reconnection can proceed at a fast rate allowing for fast dynamo action also in young galaxies.

Acknowledgements. We thank the referee Dr. James F. Drake for helpful comments. This work was supported by Polish Committee for Scientific Research (KBN) through the grant PB 404/P03/2001/20. The presented work is a continuation of a research program realized by MH under the financial support of Alexander von Humboldt Foundation.

\section{References}

Birn, J., Drake, J. F., Shay, M. A., et al. 2001, J. Geophys. Res., 106, 3715

Biskamp, D. 1986, Phys. Fluids, 29, 1520

Biskamp, D. 1996, ApSS, 242, 165

Biskamp, D., Schwarz, E., \& Drake, J. F. 1997, Phys. Plasmas, 4, 1002

Biskamp, D., \& Schwarz, E. 2001, Phys. Plasmas, 8, 4729

Kulsrud, R. M. 1999, ARA\&A, 37,37

Kulsrud, R. M. 2001, Earths, Planets, Space, 53, 417 
Kulsrud, R. M., \& Anderson, S. W 1992, ApJ, 396, 606

Lazarian, A., \& Vishniac, E. 2000, Astrophysical Plasmas: Codes, Models, and Observations, ed. J. Arthur, N. Brickhouse, \& J. Franco, RevMexAA, 9, 55

Lesch, H. 2000, Proceedings of the International School of Physcis "Enrico Fermi" Course CXLII, ed. B. Coppi, A. Ferrari, \& E. Sindoni (Amsterdam: IOS Press), 395

Parker, E. N. 1957, J. Geophys. Res., 62, 509, 1957

Petschek, H. E. 1964, The Physics of Solar Flares, AAS-NASA Symposium (NASA SP-50), ed. W. H. Hess (Greenbelt, MD: NASA), 425

Rogers, B. N., Denton, R. E., Drake, J. F., \& Shay, M. A. 2001, Phys. Rev. Lett., 87, 195004
Sato, T., \& Hayashi, T. 1979, Physics of Fluids, 22, 1189

Shay, M. A., Drake, J. F., Denton, R. E., \& Biskamp, D. 1998, J. Geophys. Res., 103, 159

Shay, M. A., \& Drake, J. F. 1998, Geophys. Res. Lett., 25, 3759

Sweet, P. A. 1958, in Electromagnetic Phenomena in Cosmic Physics, ed. B. Lehnert (Cambridge Univ. Press), 123

Tajima, T., \& Shibata, K. 1997, Plasma Astrophysics (AddisonWesley), 33

Taylor, J. B. 1986, Rev. Mod. Phys, 53, 741

Uzdensky, D., \& Kulsrud, R. M. 2000, Phys. Plasmas, 7, 4018

Vallée, J. P. 1995, Ap\&SS, 234, 1 